\title{
Correction to: Stochastic Sequential Modeling: Toward Improved Prostate Cancer Diagnosis Through Temporal-Ultrasound
}

\author{
Layan Nahlawi (iD, ${ }^{1}$ Farhad Imani, ${ }^{2}$ Mena Gaed, ${ }^{3}$ Jose A. Gomez, ${ }^{3}$ \\ Madeleine Moussa, ${ }^{3}$ Eli Gibson, ${ }^{4}$ Aaron Fenster, ${ }^{5}$ Aaron Ward, ${ }^{6}$ \\ Purang Abolmaesumi, ${ }^{2}$ Parvin Mousavi, ${ }^{1}$ and Hagit Shatkay ${ }^{1,7}$
}

${ }^{1}$ School of Computing, Queen's University, Kingston, ON K7L 2N8, Canada; ${ }^{2}$ Department of Electrical and Computer Engineering, University of British Columbia, Vancouver, BC, Canada; ${ }^{3}$ London Health Sciences Centre, London, ON, Canada; ${ }^{4}$ University College London, London, UK; ${ }^{5}$ Robarts Research Institute, London, ON, Canada; ${ }^{6}$ Department of Medical Physics, Western University, London, ON, Canada; and ${ }^{7}$ Department of Computer and Information Sciences, University of Delaware, Newark, DE, USA

(Published online 8 September 2020)

Correction to: Annals of Biomedical Engineering https://doi.org/10.1007/s10439-020-02585-y

The authors have noted an omission in the original acknowledgements. The correct acknowledgements are as follows:
Acknowledgments This work was partially supported by Grants from NSERC Discovery to Hagit Shatkay and Parvin Mousavi, NSERC and CIHR CHRP to Parvin Mousavi and NIH R01 LM012527, NIH U54 GM104941, NSF IIS EAGER \#1650851 \& NSF HDR \#1940080 to Hagit Shatkay.

Publisher's Note Springer Nature remains neutral with regard to jurisdictional claims in published maps and institutional affiliations.

Address correspondence to Layan Nahlawi, School of Computing, Queen's University, Kingston, ON K7L 2N8, Canada. Electronic mail: layan.nahlawi@queensu.ca

The original article can be found online at https://doi.org/10 1007/s10439-020-02585-y.

\section{ELECTRONIC SUPPLEMENTARY MATERIAL}

The online version of this article (doi:10.1007/s10439007-9398-3) contains supplementary material, which is available to authorized users. 